\title{
Cytotoxic evaluation of 3-aminopyridine-2-carboxaldehyde thiosemicarbazone in peripheral blood lymphocytes of patients with refractory solid tumors using electron paramagnetic resonance
}

\author{
JILL M. KOLESAR ${ }^{1}$, KAMAKSHI SACHIDANANDAM ${ }^{1}$, WILLIAM R. SCHELMAN ${ }^{1}$, JENS EICKHOFF ${ }^{2}$, \\ KYLE D. HOLEN ${ }^{1}$, ANNE M. TRAYNOR ${ }^{1}$, DONA B. ALBERTI ${ }^{1}$, JAMES P. THOMAS ${ }^{3}$, \\ CHRISTOPHER R. CHITAMBAR ${ }^{4}$, GEORGE WILDING ${ }^{1}$ and WILLIAM E. ANTHOLINE ${ }^{5}$

\begin{abstract}
${ }^{1}$ University of Wisconsin Paul P. Carbone Comprehensive Cancer Center, University of Wisconsin-Madison, Madison, WI 53792-5669; ${ }^{2}$ Department of Biostatistics, University of Wisconsin School of Medicine and Public Health, Madison, WI 53705; ${ }^{3}$ The Ohio State University Comprehensive Cancer Center, Columbus, OH 43210; ${ }^{4}$ Department of Medicine, and ${ }^{5}$ Department of Biophysics, Medical College of Wisconsin, Milwaukee, WI 53226, USA
\end{abstract}

Received September 24, 2010; Accepted November 2, 2010

DOI: $10.3892 /$ etm.2010.165

\begin{abstract}
Aminopyridine-2-carboxaldehyde thiosemicarbazone (3-AP) is a metal chelator that potently inhibits the enzyme ribonucleotide reductase (RR), which plays a key role in cell division and tumor progression. A subunit of RR has a non-heme iron and a tyrosine-free radical, which are required for the enzymatic reduction of ribonucleotides to deoxyribonucleotides. The objective of the present study was to determine whether 3-AP affects its targeted action by measuring electron paramagnetic resonance (EPR) signals formed either directly or indirectly from low molecular weight ferric-3-AP chelates. Peripheral blood lymphocytes were collected from patients with refractory solid tumors at baseline and at 2, 4.5 and $22 \mathrm{~h}$ after 3 -AP administration. Using EPR spectra, our study identified signals from high-spin Fe-transferrin, highspin heme and low-spin iron or copper ions. An increase in the Fe-transferrin signal was observed, suggesting blockage of Fe uptake. It is hypothesized that formation of reactive oxygen species by $\mathrm{FeT}_{2}$ or $\mathrm{CuT}$ damages the transferrin or the transferrin receptor. An increase in the heme signal was also observed, which was a probable source of cytochrome c release from the mitochondria and potential apoptosis. In
\end{abstract}

Correspondence to: Dr Jill M. Kolesar, University of Wisconsin Paul P. Carbone Comprehensive Cancer Center, University of Wisconsin-Madison, 600 Highland Ave., Room K4/554, Madison, WI 53792-5669, USA

E-mail: jmkolesar@pharmacy.wisc.edu

Dr William E. Antholine, Department of Biophysics, Medical College of Wisconsin, Milwaukee, WI 53226, USA

E-mail: wantholi@mcw.edu

Key words: 3-aminopyridine-2-carboxaldehyde thiosemicarbazone, electron paramagnetic resonance, ribonucleotide reductase, chelator addition, increased levels of $\mathrm{Fe}$ and $\mathrm{Cu}$ were identified. These results, which were consistent with our previous study validating 3-AP-mediated signals by EPR, provide valuable insights into the in vivo mechanism of action of 3-AP.

\section{Introduction}

3-Aminopyridine-2-carboxaldehyde thiosemicarbazone (3-AP), a metal chelator, is a potent small-molecule inhibitor of ribonucleotide reductase (RR), an enzyme that is important for cell division and tumor growth (1). The RR enzyme is responsible for reducing ribonucleotides to their corresponding deoxyribonucleotides, precursors of DNA synthesis and repair. Human $\mathrm{RR}$ is composed of two heterodimeric subunits, hRRM1, that contains the nucleotide binding site, and hRRM2, that contains the metal binding site (1). The hRRM2 subunit has a non-heme iron and a tyrosine-free radical, which are required for the enzymatic reduction of ribonucleotides (2). The RR complex contains two ferric ions that are coordinated by four carboxylates, and two histidine ligands that are anti-ferromagnetically coupled by a $\mu$-oxo bridge, rendering it electron paramagnetic resonance (EPR)-silent. Inhibitors of RR act by destroying the tyrosine-free radical in the hRRM2 subunit (3). The reaction of the di-ferric center with oxygen generates a protein-bound tyrosyl radical that is detectable by EPR (4). Recent evidence has shown that RR spontaneously loses the iron atoms, rendering it chelatable by small hydrophobic chelators, such as 3-AP (4).

Inhibition of RR disrupts DNA synthesis and repair leading to apoptotic cell death. EPR can be used to measure the effects of 3-AP in the cell. The presence of the free tyrosyl radical is directly proportional to the enzymatic activity of $\mathrm{RR}(5,6)$. 3-AP-mediated inhibition of $\mathrm{RR}$ is predicted to result in a decrease in the tyrosyl radical and an increase in the $\mathrm{Cu}$-triapine $(\mathrm{CuT})$ and iron-3-AP $\left(\mathrm{FeT}_{2}\right)$ complexes (7). In addition, 3-AP inhibits Fe uptake from transferrin and induces 
the transferrin receptor at both the levels of mRNA and protein (8-10). An increase in Fe-transferrin complexes and transferrin receptors is also expected $(9,11)$. Cytochrome c release occurs early in the apoptotic cascade, and increased cyctochrome c release is anticipated following treatment with 3-AP (12).

It has been shown that formation of the metal chelate of 3-AP and iron is essential to its cytotoxic effect, mediated by the formation of reactive oxygen species (ROS) that render RR inactive $(5,9)$. Precursors of 3-AP have been shown to sequester large quantities of iron in humans (13). The iron3-AP complex directly inhibits RR and is a step that involves hydrogen peroxide (9), much as precursors to $\mathrm{FeT}_{2}$ inhibit RR $(14,15)$. In addition, the iron-3-AP complex is redox active, forming ROS that deplete intracellular glutathione and cause DNA strand breaks, similar to iron-doxorubicin or the ironbleomycin complexes (9). Zhu et al demonstrated that 3-AP and seven of its synthetic derivatives decreased the activity of RR in a dose-dependent manner in normal as well as hyroxyurea- and gemcitabine-resistant KB cell lines, using two different forms of RR (16).

In light of recent findings, it is known that 3-AP possesses intrinsic fluorescent properties (17). Thus, by means of fluorescence microscopy, its uptake and intracellular distribution in living human cancer cells can be monitored. This feature is relatively uncommon among anticancer drugs and may help in studying resistance mechanisms by undergoing possible phenotypic changes, as well as the combinatorial effects of other drugs on 3-AP (17). 3-AP has anti-tumor effects, both in vitro and in vivo, and several clinical trials are currently being conducted to evaluate its safety and antineoplastic activity, either as monotherapy or in combination with other anticancer agents (18-22). It has also been shown that inhibition of RR by 3-AP enhances radiation-mediated cytotoxicity independent of $\mathrm{p} 53$ regulation by impairing repair processes that rely on deoxyribonucleotide production. This substantially increases the radiation sensitivity of human cancers (23). In a recent study, we validated that the effect of 3-AP on peripheral blood mononuclear cells can be measured by EPR spectroscopy (6). The goal of the present study was to determine whether 3-AP affects its targeted action in peripheral blood lymphocytes, by measuring EPR signals formed either directly or indirectly.

\section{Materials and methods}

Sample preparation. Blood samples were evaluable from 18 patients with locally advanced, unresectable or metastatic solid tumors who participated in two clinical trials at the University of Wisconsin: a phase I combination of 3-AP and doxorubicin (20), and a phase I combination of 3-AP and irinotecan (19). Peripheral blood lymphocytes (PBLs) were obtained as previously described (6). Detailed information about the dosing schedules for 3-AP, doxorubicin and irinotecan are found in the referenced literature for the respective clinical trials. Briefly, two 10-ml samples of blood were drawn into CPT tubes and were immediately centrifuged to remove the PBLs. Samples were obtained prior to administration of 3-AP and at 2, 4.5 and $22 \mathrm{~h}$ after the end of 3-AP infusion. A small aliquot of cells was removed for determining cell counts and protein concentration. The remaining PBL samples were placed in 4-mm OD quartz EPR tubes, frozen in liquid nitrogen and stored at $-80^{\circ} \mathrm{C}$ until the time of assay. Human halo-transferrin (Calbiochem; EMD Bioscienes, La Jolla, CA, USA) was dissolved in MSH (0.225 mannitol, 0.75 M sucrose and $20 \mathrm{mM}$ HEPES buffer $\mathrm{pH}$ 7.5) and used as a standard (concentration $320 \mu \mathrm{M}$ ).

EPR spectroscopy. A Varian E109 Century Series spectrometer (Varian, Palo Alto, CA, USA) operating at X-band $(9-9.5 \mathrm{GHz})$ with a $100-\mathrm{kHz}$ field modulation or a Bruker E500 ELEXSYS spectrometer with an Oxford Instruments ESR-9 helium flow cryostat and a Bruker DM0101 cavity was employed. ESR measurements were made at $10 \mathrm{~K}$ on frozen intact cells. Spectra from each sample were recorded ten times and an average was calculated.

Statistical analysis. EPR spectra recordings were taken for PBL samples collected at baseline and at 2, 4.5 and $22 \mathrm{~h}$ after administration of 3-AP. Changes in spectral parameters were evaluated by computing the ratios at 2,4.5 and $22 \mathrm{~h}$ to baseline.

The outcome variable was expressed as a ratio of the value at the given time-point (numerator of the ratio) to the baseline value (denominator of the ratio). The ratios were summarized using standard descriptive statistics in terms of number of observations, medians, ranges, means and standard deviations. Boxplots were used to display the distributions of the ratios for each time point. A non-parametric Wilcoxon Signed Rank test was used to determine statistical significance. Since the a priori hypothesis proposed that there is an increase in the Fe-transferrin signals, heme signals and $\mathrm{FE}$ and $\mathrm{Cu}$ level values from baseline, one-sided tests were used with the alternative hypotheses that the ratios are $>1$. Exact $p$-values were computed, and p-values $<0.05$ were defined as statistically significant.

\section{Results}

EPR spectra of low spin (l.s.) Fe and $\mathrm{Cu}$. EPR spectra were obtained for 3-AP upon addition of cupric or ferric ions in DMSO and PBS. This was used as a template to recognize signals in PBLs. Typical 1.s. Fe signals at $\mathrm{g}_{\mathrm{z}}=2.196, \mathrm{~g}_{\mathrm{y}}=2.138$ and $g_{x}=2.003$, and $\mathrm{Cu}$ signals at $\mathrm{g}_{11}=2.191$ and $\mathrm{A}_{11}=175 \mathrm{G}$ were obtained. These EPR parameters compared favorably to our previous validation study, as well as to the EPR parameters for $\mathrm{Fe}$ and $\mathrm{Cu}$ complexes of 2-formylpyridine monothiosemicarbazone $(6,24)$. There was a significant increase in EPR signals assigned to $\mathrm{Fe}$ and $\mathrm{Cu}$ sites at 2 and $4.5 \mathrm{~h}(\mathrm{p}=0.04$ and 0.03 ), reflective of 3 -AP binding to $\mathrm{Fe}$ or $\mathrm{Cu}$, which was not observed at $22 \mathrm{~h}$ after 3 -AP administration $(\mathrm{p}=0.29)$; this suggested that 3-AP affected its action primarily at early time-points (Fig. 1A, Table I).

EPR spectra of peripheral blood lymphocytes (PBLs), before and after treatment with 3-AP. EPR spectra of high spin (h.s.) heme were detected at $\mathrm{g}=6$. This represents the $\mathrm{Fe}(3+)$ in heme, in an oxidized state. The heme signal was amplified significantly after the administration of 3-AP, which was consistent with our previous study validating the measurement of these signals using EPR (6). There was a significant 17-fold median increase from baseline at $2 \mathrm{~h}(\mathrm{p}=0.002)$, a significant increase 
A

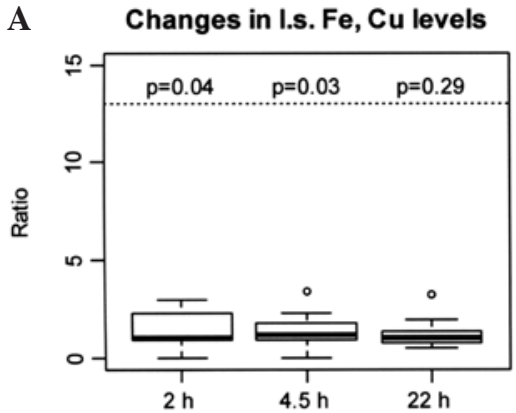

C Changes in h.s.non-heme Fe levels

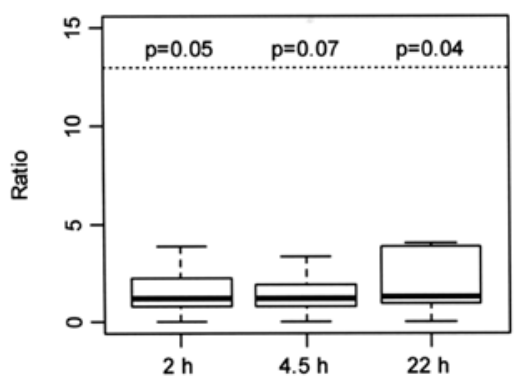

B Changes in h.s. heme signals

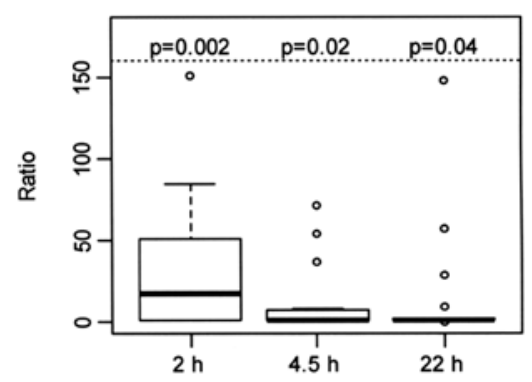

D Changes in free radicals

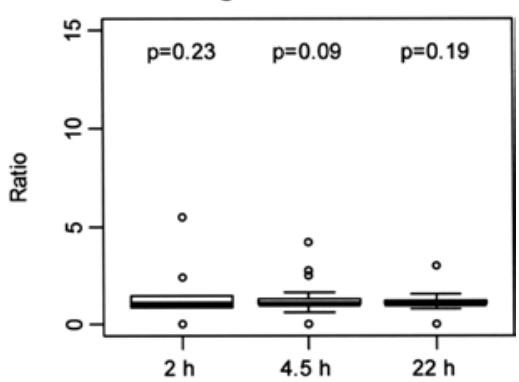

Figure 1. Box plots of ratios at 2, 4.5 and $22 \mathrm{~h}$ to baseline values. The top and bottom edges of the boxes represent the 25 th and 75 th percentiles of the data. The black line inside the box represents the median. The 5th and 95th percentiles are represented by the whiskers extending from the top and bottom of the box.

Table I. Changes in low spin Fe, $\mathrm{Cu}$ levels from baseline to 2, 4.5 and $22 \mathrm{~h}$ after 3-AP administration, expressed as ratios over baseline levels.

\begin{tabular}{lcccccc}
\hline Time & No. & Median & Range & Mean & SD & p-value \\
\hline Baseline - $2 \mathrm{~h}$ & 16 & 1.02 & $0.01-62.00$ & 5.20 & 15.17 & $0.04^{\mathrm{a}}$ \\
Baseline - 4.5 h & 18 & 1.21 & $0.01-111.0$ & 9.30 & 26.68 & $0.03^{\mathrm{a}}$ \\
Baseline - 22 h & 18 & 1.00 & $0.48-51.00$ & 3.92 & 11.77 & 0.29 \\
\hline
\end{tabular}

Values reflective of potential 3-AP binding to $\mathrm{Fe}$ or $\mathrm{Cu}$ ions. Unit of outcome variable - ratio over baseline. Baseline (denominator of ratio): value at time-point $0 .{ }^{\mathrm{a}} \mathrm{p}$-value of $<0.05$ obtained by Wilcoxon Signed Rank test (Ha ratio $>1$ ).

Table II. Changes in high spin heme signals from baseline to 2, 4.5 and $22 \mathrm{~h}$ after 3-AP administration ( $\mathrm{g}=6$ ), expressed as ratios over baseline signals.

\begin{tabular}{lcccccc}
\hline Time & No. & Median & Range & Mean & SD & p-value \\
\hline Baseline - 2 h & 16 & 16.81 & $0.67-151.0$ & 32.27 & 42.24 & $0.002^{\mathrm{a}}$ \\
Baseline - 4.5 h & 18 & 1.00 & $0.02-71.0$ & 10.55 & 20.93 & $0.020^{\mathrm{a}}$ \\
Baseline - 22 h & 18 & 1.00 & $0.02-148.0$ & 14.22 & 36.29 & $0.040^{\mathrm{a}}$ \\
\hline
\end{tabular}

Increased levels of heme after treatment suggest potential redox mechanisms involving cytochrome c release from the mitochondria. Unit of outcome variable - ratio over baseline. Baseline (denominator of ratio): value at time-point $0 .{ }^{\text {ap}}$-value of $<0.05$ obtained by Wilcoxon Signed Rank test (Ha ratio $>1)$.

at $4.5 \mathrm{~h}(\mathrm{p}=0.02)$ and a significant increase at $22 \mathrm{~h}(\mathrm{p}=0.04)$, indicating heightened heme Fe activity over a prolonged period following 3-AP administration (Fig. 1B, Table II). Although commonly hypothesized, it was difficult to establish in this case whether the heme signal was the heme in cytochrome $\mathrm{c}$ and whether it was involved in eliciting oxidative damage.
At g=4.3, EPR spectra for h.s. non-heme Fe were obtained. This signal is primarily from the $\mathrm{Fe}$ (III) in transferrin and, to a small extent, from Fe-phosphates and other non-heme Fe signals. The intensity of this signal changed with a 1.22-fold median increase at $2 \mathrm{~h}, 1.18$-fold at $4.5 \mathrm{~h}$ and 1.31 -fold at $22 \mathrm{~h}$ after 3-AP administration, although not statistically signifi- 
Table III. Changes in high spin non-heme Fe from baseline to 2, 4.5 and $22 \mathrm{~h}$ after 3-AP administration ( $\mathrm{g}=4.3$ ), expressed as ratios over baseline signals.

\begin{tabular}{lcccrr}
\hline Time & No. & Median & Range & Mean & SD \\
\hline Baseline - 2 h & 16 & 1.22 & $0.04-65.00$ & 7.98 & 18.58 \\
Baseline - 4.5 h & 18 & 1.18 & $0.04-44.00$ & 5.65 & 12.92 \\
Baseline - 22 h & 18 & 1.31 & $0.01-79.00$ & 12.03 & 25.31 \\
\hline
\end{tabular}

Increased levels of non-heme Fe, comprised mostly of Fe-transferrin signals, indicate inhibition of RR at later time-points. Unit of outcome variable - ratio over baseline. Baseline (denominator of ratio): value at time-point $0 .{ }^{a}$-value of $<0.05$ obtained by Wilcoxon Signed Rank test (Ha ratio $>1$ ).

Table IV. Changes in free radicals from baseline to 2, 4.5 and $22 \mathrm{~h}$ after 3-AP administration (g=2.005), expressed as ratios over baseline values.

\begin{tabular}{lcccrr}
\hline Time & No. & Median & Range & Mean & SD \\
\hline Baseline - 2 h & 16 & 1.00 & $0.01-49.00$ & 4.24 & 12.00 \\
Baseline - 4.5 h & 18 & 1.00 & $0.02-4.18$ & 1.35 & 0.23 \\
Baseline - 22 h & 18 & 1.00 & $0.01-120.00$ & 7.69 & 0.04 \\
\hline
\end{tabular}

Unit of outcome variable - ratio over baseline. Baseline (denominator of ratio): value at time-point $0 .{ }^{\text {a }}$-value of $<0.05$ obtained by Wilcoxon Signed Rank test (Ha ratio $>1$ ).

cant at 2 and $4.5 \mathrm{~h}(\mathrm{p}=0.05$ and 0.07$)$, but significant at $22 \mathrm{~h}$ ( $\mathrm{p}=0.04$; Fig. 1C, Table III). This Fe-transferrin signal was the oxidized state of Fe-transferrin, possibly due to the generation of ROS. These data are consistent with our previous findings in that the intensity of the peak at $\mathrm{g}=4.3$ increased at $2 \mathrm{~h}$, with a less intense signal at $4 \mathrm{~h}$. However, in our previous study there was little or no signal at $22 \mathrm{~h}$ after 3-AP administration (6). These results suggest that effective inhibition of iron uptake occurs at later time-points.

Other peaks were obtained at $\mathrm{g}=2.005$, which were attributable to free radicals. However, the difference in intensities of these peaks prior to and following the administration of 3-AP was not statistically significant (Fig. 1D, Table IV). EPR spectra for the RR tyrosyl radical were not captured (data not shown).

\section{Discussion}

Ribonucleotide reductase (RR) plays a vital role in DNA synthesis by catalyzing the conversion of nucleotides to deoxynucleotides. Without a balanced supply of deoxyribonucleotides, DNA synthesis is inhibited (1). Anticancer agents that are good chelators of iron have been sought for the purpose of inhibiting the RR enzyme by removing iron from RR (8). 3-AP is a potent inhibitor of RR (1), but it is not certain whether the inhibition is a result of removing iron from RR or whether 3-AP forms a metal complex that inhibits RR activity, or both.

In previous studies with a precursor for 3-AP, the preformed cupric complex of 2-formylpyridine monothiosemicarbazone was a potent inhibitor of RR (25). 3-AP is also a tridentate chelator that ligates Fe and other metals. Preformed Fe-3-AP is a more potent inhibitor of RR than free 3-AP (5). It is believed that 3-AP forms a complex with $\mathrm{Fe}(\mathrm{III})$, is reduced to $\mathrm{Fe}(\mathrm{II})$, generates ROS and quenches RR activity $(1,10)$. A similar scenario has been reported for the cupric complex of 2-formylpyridine monothiosemicarbazone (25).

In this study, EPR signals associated with iron and copper sites in PBLs from patients were found to increase $2 \mathrm{~h}$ after 3-AP administration and continued to increase up to $4.5 \mathrm{~h}$, with a smaller increase observed $24 \mathrm{~h}$ after treatment (Fig. 1A, Table I). A significant signal observed by EPR was that of h.s. heme iron (Fig. 1B, Table II), which was greatly increased at $2 \mathrm{~h}$ after treatment and was magnified to small extents at 4.5 and $22 \mathrm{~h}$ after 3-AP administration. This is consistent with recordings from our previous study that validated the use of EPR to capture these signals (6). One compelling hypothesis is that ROS cause cell death and release of cytochrome $\mathrm{c}$ following apoptosis. Upon release of cytochrome $\mathrm{c}$, the heme is oxidized as measured by an increase in the heme signal. Although hypothetically plausible, the heme signal is an accumulation of all oxidized hemes, and further investigation is necessary to attribute the increase in heme to cytochrome c. Nonetheless, administration of 3-AP is known to cause apoptosis (26). One of the objectives of the present study was to measure the decrease in the intensity of tyrosyl radical of RR by EPR. However, these signals could not be captured. To date, the tyrosyl radical signal from RR has only been detected in rapidly proliferating cells, i.e., $100 \%$ cancer cells.

Another signal that was unequivocally identified was the signal for Fe-transferrin, which was increased at $2 \mathrm{~h}$ after drug administration, followed by a smaller increase at $4.5 \mathrm{~h}$ and a large increase at $22 \mathrm{~h}$ after treatment (Fig. 1C, Table III). It appears that $\mathrm{Fe}$ (III)-transferrin was not reduced. It is possible that $\mathrm{Fe}$ uptake was blocked, probably through the generation 
of ROS from $\mathrm{FeT}_{2}$ or $\mathrm{CuT}$, which damaged transferrin or the transferrin receptor. In support of this idea, it is known that 2-formylpyridine monothiosemicaboxylate $\mathrm{Cu}$ (II) inhibits cellular iron uptake in addition to inhibiting RR (11). Assuming that ROS are generated by either $\mathrm{FeT}_{2}$, CuT or adducts of these complexes that form either by replacing $\mathrm{T}$ or by occupying the open equatorial site of the tri-dentate cupric complex, other sites may also be damaged. The Fe-3-AP complex has been shown to dramatically increase ROS production in model systems (9).

Of note, the pharmacokinetic parameters of 3-AP obtained from phase I clinical trial data demonstrate a Tmax of $0.04 \pm 0.11 \mathrm{~h}$ in erythrocytes and $0.22 \pm 0.11 \mathrm{~h}$ in plasma $(19,20)$. These peak concentrations appear much earlier than the observed pharmacodynamic effects of 3-AP at 2, 4.5 and $22 \mathrm{~h}$ depicted in Fig. 1. This delayed effect could be due to a number of factors, such as slow uptake from plasma and a prolonged intracellular retention of 3-AP prior to formation of metal complexes, which would suggest a slow manifestation of cytotoxicity and potential apoptosis. Further studies are required to determine the reasons for this delayed effect of 3 -AP in relation to its pharmacokinetics.

In conclusion, this study provides novel insight into EPR evaluation of the effects of 3-AP in patients, identifying an $\mathrm{Fe}$-transferrin signal and potential cytochome $\mathrm{c}$ release from mitochondria, in addition to detecting signals at the $\mathrm{g}=2$ region. Formation of metal complexes of 3-AP are likely essential to its anticancer effect, whether the mechanism is related to RR inhibition or the formation of ROS, or both $(1,10)$. These results provide direct evidence for the formation of metal complexes, although not assigned, in patients receiving 3-AP therapy and provide valuable insight into the in vivo mechanism of the action of 3-AP.

\section{Acknowledgements}

This study was supported by the U01 CA62491, CTEP Translational Research Initiative Funds 24XS090, the NIH GCRC Grant M01 RR03186 and the NIH-National Biomedical ESR Center EB001980 Grant.

\section{References}

1. Yu Y, Wong J, Lovejoy D, Kalinowski D and Richardson D: Chelators at the cancer coalface: desferrioxamine to Triapine and beyond. Clin Cancer Res 12: 6876-6883, 2006.

2. Nordlund P, Sjöberg B and Eklund H: Three-dimensional structure of the free radical protein of ribonucleotide reductase. Nature 345: 593-598, 1990.

3. Qiu W, Zhou B, Darwish D, Shao J and Yen Y: Characterization of enzymatic properties of human ribonucleotide reductase holoenzyme reconstituted in vitro from hRRM1, hRRM2, and p53R2 subunits. Biochem Biophys Res Commun 340: 428-434, 2006.

4. Cooper C, Lynagh G, Hoyes K, Hider R, Cammack R and Porter J: The relationship of intracellular iron chelation to the inhibition and regeneration of human ribonucleotide reductase. J Biol Chem 271: 20291-20299, 1996.

5. Shao J, Zhou B, Di Bilio A, et al: A Ferrous-Triapine complex mediates formation of reactive oxygen species that inactivate human ribonucleotide reductase. Mol Cancer Ther 5: 586-592, 2006.

6. Kolesar J, Schelman W, Geiger P, et al: Electron paramagnetic resonance study of peripheral blood mononuclear cells from patients with refractory solid tumors treated with Triapine. J Inorg Biochem 102: 693-698, 2008.
7. Petering DH and Antholine WE: Reviews in Biochemical Toxicology. Vol 9. Hodgson E, Bend JR and Philpot RM (eds). Elsevier, New York, 1988.

8. Richardson D, Sharpe P, Lovejoy D, et al: Dipyridyl thiosemicarbazone chelators with potent and selective antitumor activity form iron complexes with redox activity. J Med Chem 49: 6510-6521, 2006.

9. Whitnall M, Howard J, Ponka P and Richardson D: A class of iron chelators with a wide spectrum of potent antitumor activity that overcomes resistance to chemotherapeutics. Proc Natl Acad Sci USA 103: 14901-14906, 2006.

10. Chaston T, Lovejoy D, Watts R and Richardson D: Examination of the antiproliferative activity of iron chelators: multiple cellular targets and the different mechanism of action of Triapine compared with desferrioxamine and the potent pyridoxal isonicotinoyl hydrazone analogue 311. Clin Cancer Res 9: 402-414, 2003.

11. Narasimhan J, Antholine W, Chitambar C and Petering D: Inhibition of iron uptake in HL60 cells by 2-formylpyridine monothiosemicarbazonato $\mathrm{Cu}(\mathrm{II})$. Arch Biochem Biophys 289: 393-398, 1991.

12. Martinou J, Desagher S and Antonsson B: Cytochrome c release from mitochondria: all or nothing. Nat Cell Biol 2: E41-E43, 2000.

13. DeConti R, Toftness B, Agrawal K, et al: Clinical and pharmacological studies with 5-hydroxy-2-formylpyridine thiosemicarbazone. Cancer Res 32: 1455-1462, 1972.

14. Sartorelli A: Effect of chelating agents upon the synthesis of nucleic acids and protein: inhibition of DNA synthesis by 1-formylisoquinoline thiosemicarbazone. Biochem Biophys Res Commun 27: 26-32, 1967.

15. Sartorelli A, Agrawal K and Moore E: Mechanism of inhibition of ribonucleoside diphosphate reductase by a-(N)heterocyclic aldehyde thiosemicarbazones. Biochem Pharmacol 20: 3119-3123, 1971.

16. Zhu L, Zhou B, Chen X, Jiang H, Shao J and Yen Y: Inhibitory mechanisms of heterocyclic carboxaldehyde thiosemicabazones for two forms of human ribonucleotide reductase. Biochem Pharmacol 78: 1178-1185, 2009.

17. Kowol C, Trondl R, Arion V, Jakupec M, Lichtscheidl I and Keppler B: Fluorescence properties and cellular distribution of the investigational anticancer drug Triapine (3-aminopyridine2-carboxaldehyde thiosemicarbazone) and its zinc(II) complex. Dalton Trans 39: 704-706, 2010.

18. Attia S, Kolesar J, Mahoney M, et al: A phase 2 consortium (P2C) trial of 3-aminopyridine-2-carboxaldehyde thiosemicarbazone (3-AP) for advanced adenocarcinoma of the pancreas. Invest New Drugs 26: 369-379, 2008.

19. Choi B, Alberti D, Schelman W, et al: The maximum tolerated dose and biologic effects of 3-aminopyridine-2-carboxaldehyde thiosemicarbazone (3-AP) in combination with irinotecan for patients with refractory solid tumors. Cancer Chemother Pharmacol 66: 973-980, 2010.

20. Schelman W, Morgan-Meadows S, Marnocha R, et al: A phase I study of Triapine in combination with doxorubicin in patients with advanced solid tumors. Cancer Chemother Pharmacol 63: 1147-1156, 2009.

21. Nutting C, van Herpen C, Miah A, et al: Phase II study of 3-AP Triapine in patients with recurrent or metastatic head and neck squamous cell carcinoma. Ann Oncol 20: 1275-1279, 2009.

22. Traynor A, Lee J, Bayer G, et al: A phase II trial of Triapine (NSC\# 663249) and gemcitabine as second line treatment of advanced non-small cell lung cancer: Eastern Cooperative Oncology Group Study 1503. Invest New Drugs 28: 91-97, 2010.

23. Kunos C, Chiu S, Pink J and Kinsella T: Modulating radiation resistance by inhibiting ribonucleotide reductase in cancers with virally or mutationally silenced p53 protein. Radiat Res 172 : 666-676, 2009.

24. Antholine W, Knight J, Whelan H and Petering D: Studies of the reaction of 2-formylpyridine thiosemicarbazone and its iron and copper complexes with biological systems. Mol Pharmacol 13: 89-98, 1977.

25. Saryan L, Mailer K, Krishnamurti C, Antholine W and Petering D: Interaction of 2-formylpyridine thiosemicarbazonato copper (II) with Ehrlich ascites tumor cells. Biochem Pharmacol 30: 1595-1604, 1981.

26. Alvero A, Chen W, Sartorelli A, Schwartz P, Rutherford T and Mor G: Triapine (3-aminopyridine-2-carboxaldehyde thiosemicarbazone) induces apoptosis in ovarian cancer cells. J Soc Gynecol Investig 13: 145-152, 2006. 